\title{
A NOTE ON THE SEPARATION OF CONNECTED SETS BY FINITE SETS
}

\author{
C. E. BURGESS
}

A connected set $K$ is said to be separated by a subset $H$ of $K$ if $K-H$ is not connected. J. R. Kline has shown that if $n$ is an integer greater than two and the plane continuum $M$ is separated by every subset of $M$ consisting of $n$ points, then $M$ is separated by some set consisting of $n-1$ points [1, Theorem 5]. A stronger conclusion has been obtained by G. T. Whyburn for the case where $M$ is a locally compact connected metric space. In fact, it follows from Whyburn's results that if every set consisting of $n$ points separates the nondegenerate locally compact connected metric space $M$, then $M$ is a Menger regular curve and contains uncountably many mutually exclusive pairs of points each pair of which separates $M[2, \mathrm{p} .313]$. It is the purpose of this note to present a proof of a related theorem for a connected topological space.

THEOREM. If $S$ is a nondegenerate connected topological space ${ }^{1}$ and $D$ is an open set such that each infinite subset of $D$ contains a finite set that separates $S$, then some pair of points in $D$ separates $S$.

The following two lemmas will be used in the proof of this theorem.

Lemma 1. If $S$ is a connected topological space, $M_{1}$ and $M_{2}$ are mutually exclusive closed sets such that $M_{2}$ does not separate $S$, and $H$ is a connected subset of $S-\left(M_{1}+M_{2}\right)$ such that some open set contains $M_{1}$ and lies in $H+M_{1}$, then $M_{1}+M_{2}$ does not separate $S$.

Proof. Suppose $S-\left(M_{1}+M_{2}\right)$ is the sum of two mutually separated sets $X$ and $Y$, where $X$ contains the connected set $H$. Since some open set lies in $H+M_{1}$ and contains $M_{1}$, it follows that no point of $M_{1}$ is a limit point of $Y$. This leads to the contradiction that $S-M_{2}$ is the sum of the two mutually separated sets $X+M_{1}$ and $Y$.

Lemma 2. If $D$ is an open set in a connected topological space $S, L$ is a finite subset of $D$ consisting of more than two points such that $S-L$ is the sum of two mutually separated sets $H$ and $K$, and no subset of $D$ with fewer points than $L$ separates $S$, then for each point $p$ of $D \cdot H$ the set $H+L-p$ is connected.

Presented to the Society, September 2, 1955; received by the editors July 22, 1955 and, in revised form, January 16, 1956.

1 The definition of a topological space given in [3] is used here. 
Proof. Suppose there is a point $p$ of $D \cdot H$ such that $H+L-p$ is the sum of two mutually separated sets $X$ and $Y$. Since $S-P$ is connected, it follows that both $X$ and $Y$ intersect $L$. Let $n$ denote the number of points in $L$. Since $n>2$, it follows that one of the sets $X \cdot L+p$ and $Y \cdot L+p$ consists of less than $n$ points. This involves a contradiction since each of these two subsets of $D$ separates $S$.

Proof of THEOREM. Suppose that no pair of points in $D$ separates $S$. Let $L_{1}$ be a subset of $D$ such that (1) $S-L_{1}$ is the sum of two mutually separated sets $H_{1}$ and $K_{1}$ and (2) no set in $D$ with fewer points than $L_{1}$ separates $S$. Let $p_{1}$ be a point of $K_{1} \cdot D$. By Lemma 2, $K_{1}+L_{1}-p_{1}$ is connected.

Let $L_{2}$ be a subset of $D \cdot H_{1}$ such that (1) $S-L_{2}$ is the sum of two mutually separated sets $H_{2}$ and $K_{2}$, where $K_{2}$ contains the connected set $K_{1}+L_{1}$, and (2) no set in $D \cdot H_{1}$ with fewer points than $L_{2}$ separates $S$. Let $p_{2}$ be a point of $D \cdot\left[K_{2}-\left(K_{1}+L_{1}\right)\right]$. By Lemma $2, K_{2}+L_{2}-p_{2}$ is connected, and since $K_{1}+L_{1}-p_{1}$ is connected and $K_{1}$ is an open set lying in $K_{1}+L_{1}$, it follows from Lemma 1 that $p_{1}+p_{2}$ does not separate the connected set $K_{2}+L_{2}$.

Let $L_{3}$ be a subset of $D \cdot H_{2}$ such that (1) $S-L_{3}$ is the sum of two mutually separated sets $H_{3}$ and $K_{3}$, where $K_{3}$ contains the connected set $K_{2}+L_{2}$, and (2) no set in $D \cdot H_{2}$ with fewer points than $L_{3}$ separates $S$. Let $p_{3}$ be a point of $D \cdot\left[K_{3}-\left(K_{2}+L_{2}\right)\right]$. By Lemma $2, K_{3}+L_{3}-p_{3}$ is connected, and since $K_{2}+L_{2}-\left(p_{1}+p_{2}\right)$ is connected and $K_{2}$ is an open set lying in $K_{2}+L_{2}$, it follows from Lemma 1 that $p_{1}+p_{2}+p_{3}$ does not separate the connected set $K_{3}+L_{3}$.

By continuing this process indefinitely, a sequence of distinct points $p_{1}, p_{2}, p_{3}, \cdots$ of $D$ can be obtained such that, for each $n$, $p_{1}+p_{2}+\cdots+p_{n}$ does not separate the connected set $K_{n}+L_{n}$. Since each $H_{n}+L_{n}$ is connected, it readily follows that for each $n, p_{1}$ $+p_{2}+\cdots+p_{n}$ does not separate $S$. This leads to the contradiction that no finite subset of the infinite set $p_{1}+p_{2}+p_{3}+\cdots$ separates $S$.

CoROLlaRY. If $n$ is a positive integer and the nondegenerate connected topological space $S$ is separated by every set consisting of $n$ points, then each open set contains a pair of points that separates $S$.

\section{REFERENCES}

1. J. R. Kline, Closed connected sets which are disconnected by the removal of a finite number of points, Proc. Nat. Acad. Sci. U.S.A. vol. 9 (1923) pp. 7-12.

2. G. T. Whyburn, Local separating points of continua, Monatshefte für Mathematik und Physik vol. 36 (1929) pp. 305-314. 1942.

3. - Analytic topology, Amer. Math. Soc. Colloquium Publications, vol. 28,

UNIVERSITY OF UTAH 